\title{
Prueba de patrones de frecuencia y patrones de duración: Evaluación del ordenamiento auditivo temporal
}

\author{
Frequency pattern and duration pattern test: \\ Assessment of auditory temporal ordering
}

Anthony Marcotti F', Sebastián Rivera $\mathbf{R}^{2}$.

\begin{abstract}
RESUMEN
Los aspectos temporales de la audición se consideran unos de los mecanismos claves del procesamiento auditivo, ya que resultarían críticos para el adecuado funcionamiento del resto de los procesos auditivos centrales. El ordenamiento auditivo temporal es una de las cuatro habilidades de los aspectos temporales de la audición y se refiere al procesamiento de dos o más estímulos auditivos según su orden de aparición u ocurrencia en el dominio temporal. Ha sido uno de los procesos más ampliamente estudiados debido a sus implicancias en el resto de las habilidades auditivas, así como también en numerosas actividades de la vida diaria, incluyendo la percepción y la discriminación de los sonidos del habla. Históricamente se han utilizado dos pruebas para evaluarlo: la prueba de patrones de frecuencia y la prueba de patrones de duración. Ambas pruebas cuentan con buena sensibilidad y especificidad para detectar lesiones del sistema nervioso auditivo central, incluyendo el hemisferio derecho, izquierdo y cuerpo calloso. En la actualidad, ambas pruebas son utilizadas con frecuencia debido a su eficiencia, su facilidad para ser administrada y la disponibilidad de valores normativos para un amplio rango de población. Se recomienda ampliamente su utilización en la práctica clínica considerando la obtención de valores normativos locales.
\end{abstract}

Palabras clave: Audición, ordenamiento auditivo temporal, prueba de patrones de frecuencia, prueba de patrones duración.

\begin{abstract}
The temporal aspects of audition are considered one of the key mechanisms of auditory processing, as they would be critical for the proper functioning of the rest of the central auditory processes. The auditory temporal ordering is referred to one of the four skills of the temporal aspects of audition and refers to the processing of two or more auditory stimuli in their order of appearance or occurrence in the time domain. It has been
\end{abstract}

1 Facultad de Ciencias de la Salud, Universidad de las Américas, Providencia.

2 Facultad de Salud, Universidad Santo Tomás, Viña del Mar.

Recibido el 14 de noviembre, 2016. Aceptado el 6 de febrero, 2017. 
one of the most widely studied processes due to its implications for the rest of listening skills, as well as in numerous activities of daily life, including perception and discrimination of speech sounds. Historically, two test has been used to evaluate: the frequency pattern and duration pattern tests. Both tests have good sensitivity and specificity to detect lesions at the central auditory nervous system, such as right and left hemisphere and corpus callosum dysfunctions. Currently, both tests are commonly used clinically due to its efficiency, ease of administration and the availability of normative data in wide range of population. Their use is strongly recommended in clinical practice considering obtaining local normative values.

Key words: Auditory temporal ordering, frequency pattern test, duration pattern test.

\section{INTRODUCCIÓN}

El procesamiento auditivo (central) o $\mathrm{PA}(\mathrm{C})$, se refiere a la eficiencia y eficacia con que el sistema nervioso central (SNC) utiliza la información auditiva1. De manera más precisa, el $\mathrm{PA}(\mathrm{C})$ hace referencia al procesamiento perceptual de la información auditiva en el SNC y a la actividad neurobiológica en el cual se sustenta dicho procesamiento, la cual se puede analizar mediante Ios potenciales auditivos electrofisiológicos. El $\mathrm{PA}(\mathrm{C})$ incluye los mecanismos auditivos que sustentan las siguientes habilidades ${ }^{1-3}$ : Iocalización y lateralización sonora, discriminación auditiva, reconocimiento de patrones auditivos, aspectos temporales de la audición, desempeño auditivo con señales acústicas competitivas y desempeño auditivo con señales acústicas degradadas.

Los aspectos temporales de la audición se definen como los procesos implicados en la percepción del sonido y de sus variaciones en un dominio temporal restringido o bien delimitado ${ }^{4}$. Se consideran dentro de los mecanismos claves del procesamiento auditivo, ya que resultarían críticos para el adecuado funcionamiento del resto de las habilidades, sin embargo, sus mecanismos neuronales no se encuentran del todo claros. Estos aspectos temporales se detallan en la Tabla $1 \mathrm{y}$ corresponden a: ordenamiento o secuenciación temporal, resolución o discriminación temporal, integración o sumación temporal y enmascaramiento temporal. Uno de los más estudiados ha sido el ordenamiento temporal, ya que de él dependen una serie de tareas y actividades auditivas de la vida diaria. Por ejemplo, la percepción de una melodía musical depende de la habilidad de percibir el orden de una serie de notas musicales y acordes, y de la habilidad para identificar si las frecuencias de éstas son ascendentes o descendentes con respecto a otras notas y acordes adyacentes ${ }^{5}$.

En la percepción del habla, el procesamiento temporal es una de las funciones necesarias para la discriminación de algunas claves lingüísticas, como ocurre con la discriminación de los fonemas con un mismo punto y modo articulatorio, pero que se diferencian sólo en la vibración de los pliegues vocales (por ejemplo, el fonema $/ \mathrm{p} / \mathrm{del} / \mathrm{b} /$, el fone$\mathrm{ma} / \mathrm{t} / \mathrm{del} / \mathrm{d} / \mathrm{y}$ el fonema $/ \mathrm{k} / \mathrm{del} / \mathrm{g} /$ ). Otro ejemplo

Tabla 1. Aspectos temporales de la audición

\begin{tabular}{|ll|}
\hline Aspecto & Definición \\
\hline Ordenamiento Temporal & $\begin{array}{l}\text { Procesamiento de dos o más estímulos auditivos según su orden de aparición u ocurrencia en el dominio } \\
\text { temporal. }\end{array}$ \\
$\begin{array}{l}\text { Resolución Temporal } \\
\text { Integración Temporal }\end{array}$ & $\begin{array}{l}\text { Sumación de la actividad neuronal en función de la duración de un estímulo acústico y que produce una } \\
\text { mejora en los umbrales auditivos. }\end{array}$ \\
Enmascaramiento Temporal & $\begin{array}{l}\text { Presentación no simultánea entre una señal objetivo y un ruido enmascarante, pudiendo este último apare- } \\
\text { cer antes (enmascaramiento anterógrado) o después (enmascaramiento retrógrado) de la señal. }\end{array}$ \\
\hline
\end{tabular}

Definiciones extraídas de Musiek, F. \& Chermak, G. (2015) ${ }^{75}$. 
de la importancia del procesamiento temporal, es la discriminación de palabras similares, en donde el orden o la secuencia de los fonemas que la conforman y la discriminación de la duración entre estos mismos fonemas es fundamental2. Es más, el procesamiento de algunas claves acústicas de los sonidos del habla depende de la percepción de la frecuencia y de la duración del estímulo como una secuencia de eventos ${ }^{3}$.

\section{ORDENAMIENTO TEMPORAL AUDITIVO}

El ordenamiento temporal, o también llamado en ocasiones secuenciación temporal, se refiere al procesamiento de dos o más estímulos auditivos según su orden de aparición u ocurrencia en el dominio temporal ${ }^{6}$. Esta categoría ha sido ampliamente estudiada, principalmente por su importancia en la percepción del habla ${ }^{7-9}$. Individuos que tienen dificultades reconociendo patrones temporales tienen además dificultades para extraer y utilizar los aspectos prosódicos del habla, como el ritmo, velocidad y entonación, lo que impide al oyente identificar la intencionalidad del interlocutor, interpretar la ironía y el sarcasmo ${ }^{10}$. El ordenamiento temporal es un proceso complejo, y aunque requiere mucho más que la secuenciación de los eventos acústicos, este sería su componente primario.

La habilidad para reconocer, identificar y secuenciar patrones auditivos de manera adecuada, depende de una serie de procesos, tanto perceptuales como cognitivos ${ }^{6}$. Estos procesos no son restrictivos a un solo hemisferio, sino que requieren de la integración de la información de ambos a través del cuerpo calloso ${ }^{11}$. Antes de los estudios en pacientes epilépticos sometidos a una callosotomía, se asumía que el lóbulo temporal del hemisferio izquierdo era el principal responsable para la secuenciación temporal auditiva ${ }^{12}$. Efron ${ }^{13}$ sugirió inicialmente, que a pesar que el procesamiento temporal es inconsciente y se lleva a cabo en todo nivel a través del sistema nervioso, el análisis del orden temporal de dos estímulos se lleva a cabo de manera primaria en el hemisferio dominante del sujeto, específicamente en el lóbulo temporal, extendiéndose posteriormente hasta el área de Wernicke (área 22 de Brodmann). Sin embargo, está bien establecido desde hace varios años que ninguno de los dos hemisferios de manera independiente puede procesar adecuadamente los patrones temporales ${ }^{11,14}$. Por lo tanto, un ordenamiento temporal adecuado requiere que tanto el hemisferio derecho como izquierdo estén anatómicamente y fisiológicamente intactos, y conectados eficientemente a través del cuerpo calloso.

Existe evidencia que la corteza auditiva es responsable de no solo la organización de los eventos neurales, sino que además de mantener la secuencia adecuada del estímulo acústico ${ }^{15}$. Sin embargo, a diferencia de la percepción del orden temporal de manera independiente de dos o más estímulos, la verbalización de dicho orden (patronaje temporal) es una tarea más compleja. Pinheiro y Ptacek ${ }^{16}$ desarrollaron una prueba de procesamiento temporal de ordenamiento y etiquetado verbal de tres sonidos de distinta frecuencia. Los mecanismos subyacentes a esta tarea fueron explicados años más tarde por Musiek y cols ${ }^{11}$ basados en el procesamiento dominante de cada hemisferio cerebral. El hemisferio no dominante (generalmente el derecho) participa en la percepción de la altura tonal17,18, al igual que en el reconocimiento del contorno musical y en las formas de los patrones $^{19,20}$. De hecho, se considera que el hemisferio derecho es responsable en mayor medida de la prosodia, que es el componente del lenguaje que refiere al procesamiento cognitivo necesario para comprender 0 expresar intenciones comunicativas usando aspectos suprasegmentales del habla, tales como las variaciones de la entonación, las pausas y las modulaciones de la intensidad vocal, que corresponden en su mayoría a aspectos temporales $^{21}$. Las bases neurales para la percepción de la frecuencia no se conocen del todo, sin embargo, se asume que las regiones corticales auditivas del hemisferio derecho tienen una mayor resolución frecuencial en comparación con las áreas homólogas del hemisferio izquierdo ${ }^{22}$.

El hemisferio dominante, que es generalmente el izquierdo en al menos el $80 \%$ de personas tanto zurdas como diestras ${ }^{23,24}$, participa cuando es necesaria la verbalización de las respuestas ${ }^{25}$. El área sensitiva del lenguaje corresponde específicamente al área de Wernicke, que está conectada con el área de Broca (área 44 y 45 de Brodmann) por un haz de fibras nerviosas denominado fascículo ar- 
queado, por lo que permite no solo la comprensión del lenguaje, sino que además su expresión ${ }^{26}$. Por ende, el etiquetado verbal de los patrones tonales utilizados en esta tarea requiere, en primer lugar, el procesamiento del contorno acústico por el hemisferio derecho, luego transferirlo vía cuerpo calloso al hemisferio izquierdo, para finalmente ser etiquetado verbalmente. No se tiene claridad en relación a estos mecanismos fisiológicos en sujetos con hemisferio derecho dominante ${ }^{27}$. Sin embargo, está bastante claro que ciertas lesiones en el hemisferio izquierdo pueden causar alteraciones severas de lenguaje, $y$, aunque el hemisferio derecho se relaciona principalmente con aspectos paralingüísticos (entonación, intensidad, velocidad, ritmo y pausas), también podrían producirse alteraciones lingüísticas más sutiles como, por ejemplo, en el procesamiento léxico-semántico ${ }^{28-29}$, habilidades pragmáticas $^{30}$ y habilidades discursivas ${ }^{31}$.

A pesar que el ser humano necesita periodos de tiempo relativamente cortos entre un estímulo y otro para identificar la presencia de ambos, se necesitan tiempos mayores para poder identificar su orden de presentación de manera adecuada. La resolución, o también llamada discriminación temporal, se refiere a la mínima cantidad de tiempo en la cual un individuo puede discriminar entre dos señales auditivas ${ }^{32}$. Para sujetos con audición normal, este intervalo de tiempo interestímulo (ITI), es generalmente de 2 a $3 \mathrm{~ms}^{33}$. Hirsh ${ }^{5}$ estudió el efecto de los ITI en la percepción del ordenamiento temporal. Utilizando una variedad de estímulos acústicos, determinó que se requiere un ITI de alrededor de $17 \mathrm{~ms}$ para que el oyente sea capaz de identificar cuál de los dos estímulos aparece primero con al menos $75 \%$ de precisión. Este último correspondería al umbral de ordenamiento temporal, definido como el mínimo intervalo de tiempo entre dos estímulos, necesario para que una secuencia o patrón sea reconocible ${ }^{34}$.

De manera paralela, se ha explorado el efecto de la modalidad sensorial en la percepción del orden temporal ${ }^{35}$. Se ha descrito que se requieren de ITI de aproximadamente $20 \mathrm{~ms}$ para reportar un orden temporal, con una precisión $75 \%$, sin importar si el estímulo es acústico, táctil, visual 0 combinados en modalidad. Estos resultados sugieren que existiría a nivel cerebral, algún tipo de sistema de organización temporal que es independiente de
Ios sistemas periféricos y/o centrales de modalidad específica. De manera similar, se ha demostrado que algunos niños con alteraciones de audición, lenguaje y/o lectoescritura, tienen problemas para procesar estímulos rápidos o de corta duración, independiente de la modalidad sensoria| ${ }^{36,37}$. Este disturbio multisensorial sugiere la existencia de un sistema de organización de modalidad no específica en el cerebro humano. Además, al igual como han planteado otros autores ${ }^{1,38,39}$, esto apoyaría tempranamente la idea que la modalidad específica completa como criterio diagnóstico para un desorden del procesamiento auditivo (DPA), al menos desde algunos aspectos temporales, sería neurofisiológicamente insostenible.

La habilidad para secuenciar una serie de estímulos auditivos no solamente depende de un ITI adecuado. Algunas otras variables también pueden influir de manera significativa en esta habilidad como, por ejemplo, la experiencia previa del sujeto o su entrenamiento auditivo, el tipo de estímulo utilizado, el número de estímulos presentados, la duración de los estímulos y la forma de presentación de los estímulos ${ }^{6}$. Efron ${ }^{13}$ demostró una diferencia significativa en el desempeño de pruebas temporales entre sujetos con y sin entrenamiento previo, lo cual sería esperable para casi todas las pruebas de procesamiento temporal, ya que se tratarían de procesos altamente entrenables ${ }^{4}$. También es importante el tipo de estímulo utilizado, por lo que se han utilizado distintos tipos de ruido, tonos, clics e incluso estímulos verbales. Quizás una de las consideraciones más críticas es el efecto de la integración temporal, como se refleja en la duración de los estímulos. Si se utilizan estímulos de menos de 200 ms de duración, el desempeño en las pruebas se verá afectado en relación a la utilización de estímulos de más de 200 ms de duración ${ }^{40}$. Este fenómeno sería igualmente válido para la tasa de presentación de los estímulos, donde los ITI más cortos, producirían un desempeño más deficiente ${ }^{41}$.

\section{PRUEBA DE PATRONES DE FRECUENCIA}

Clásicamente, en la práctica clínica se han utilizado dos pruebas de percepción de patrones auditivos para evaluar alteraciones del procesamiento auditivo 
temporal y lesiones en el sistema nervioso auditivo central (SNAC). Una de ellas es la prueba de patrones de frecuencia, que fue descrita inicialmente por Pinheiro y Ptacek ${ }^{16}$. Fue adaptada para la práctica clínica unos años más tarde ${ }^{42}$ y actualmente es una de las pruebas clínicas que conforman la batería para la evaluación del procesamiento auditivo más ampliamente utilizadas, probablemente debido a su eficiencia y a su facilidad para ser administrada ${ }^{43,44}$. Esta prueba consta originalmente de 120 secuencias (60 para cada oído), cada una compuesta por una serie de tres tonos puros, que pueden ser de $1.122 \mathrm{~Hz} 0$ de $880 \mathrm{~Hz}^{45}$. La tarea del paciente es indicar verbalmente los tres tonos, en el mismo orden en el cual fueron presentados. De esta manera, los tonos de 880 y $1.122 \mathrm{~Hz}$ deben ser verbalizados como tonos "bajos" o "graves" (B) y "altos" o "agudos" (A) respectivamente. Existen seis posibles combinaciones aleatorias ( $A A B$, $B B A, A B A, B A B, B A A$ y $A B B)$, sin que en ninguna combinación se repita tres veces el mismo tono. Cada tono tiene una duración de 150 ms con un ITI de 200 ms (Figura 1). Se recomienda que esta prueba sea presentada a $50 \mathrm{~dB} \mathrm{HL}$, sin embargo, esto no es crítico y puede ser modificado según la audición de cada paciente. Por lo general el máximo desempeño en esta prueba se obtiene alrededor de los 10 a $15 \mathrm{~dB}$ SL46, por lo que también se recomienda aplicarla entre los 20 a $50 \mathrm{~dB} \mathrm{SL}, 020$ a $50 \mathrm{~dB}$ sobre el promedio tonal puro o PTP (promedio de los umbrales auditivos aéreos en las frecuencias 0,5, 1 y $2 \mathrm{kHz})^{4}$. Cabe destacar que también son aceptadas otras modalidades de respuesta: que el paciente tararee los tonos escuchados 0 , en el caso de los niños más pequeños, que indiquen una figura que represente las características del sonido escuchado ${ }^{46}$.

Debido a que la prueba puede ser demasiado larga para algunos pacientes, o los resultados de-

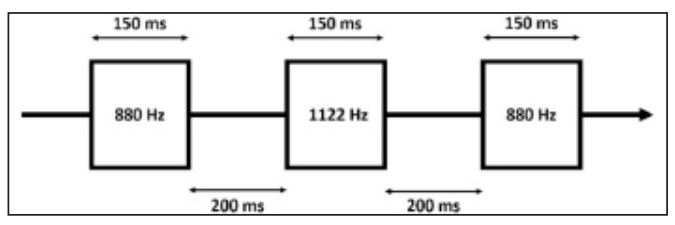

Figura 1. Ejemplo de patrones de frecuencia. Presentación de tres tonos ( 880 y $1122 \mathrm{~Hz}$ ) de una duración de $150 \mathrm{~ms}$ y con un ITI de $200 \mathrm{~ms}$. masiado evidentes a simple vista para la aplicación de todas las secuencias, existen algunas formas de simplificar la prueba y hacerla más amigable clínicamente. En primer lugar, es posible aplicar solamente 30 en vez de 60 secuencias en cada oído ${ }^{45}$. Además, la prueba se puede dar por finalizada si el paciente logra responder de forma correcta al menos 14 de las 15 primeras secuencias de la prueba. Por último, si el sujeto evaluado no logra responder de manera correcta 14 de las 15 primeras secuencias, la prueba igualmente puede darse por terminada ${ }^{46}$. Las omisiones, reversiones 0 errores en los patrones son consideradas respuestas incorrectas ${ }^{45}$ y los resultados se expresan en porcentajes de respuestas correctas.

La prueba de patrones de frecuencia puede detectar alteraciones del procesamiento temporal, incluso en menores con dificultades del aprendiza$\mathrm{je}^{47,48}$. Además, tiene una sensibilidad de $83 \%$ para detectar lesiones cerebrales, $45 \%$ de sensibilidad para lesiones a nivel de tronco cerebral y es relativamente resistente a hipoacusias sensorioneurales leves y moderadas, demostrando una especificidad de $88 \%{ }^{49}$.

\section{PRUEBA DE PATRONES DE DURACIÓN}

La prueba de patrones de duración fue desarrollada por Musiek y sus cols ${ }^{50}$. Al igual que la prueba de patrones de frecuencia, es una de las pruebas de evaluación clínica para detectar alteraciones del procesamiento tempora ${ }^{43,44}$. Esta prueba consta originalmente de 120 secuencias (60 para cada oído), cada una compuesta por una serie de tres tonos puros de $1 \mathrm{kHz}$ separados por un ITI de 300 ms. Estos tonos varían en su duración, pudiendo ser de 250 ms o de 500 ms (Figura 2). La tarea del paciente es indicar la duración de los tres tonos,

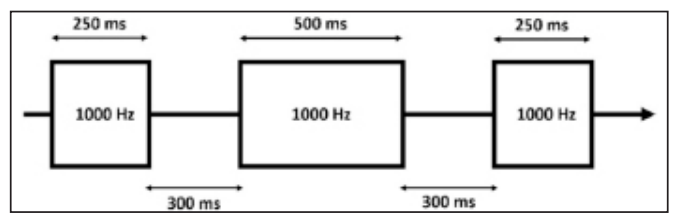

Figura 2. Ejemplo de patrones de frecuencia. Presentación de tres tonos $(1 \mathrm{kHz})$ de una duración de 250 y 500 ms y con un ITI de $300 \mathrm{~ms}$. 
en el mismo orden en el cual fueron presentados. De esta manera, los tonos de $250 \mathrm{~ms}$ deben ser verbalizados como tonos "cortos" (C) y los tonos de $500 \mathrm{~ms}$ deben ser verbalizados como tonos "largos" (L). Existen seis posibles combinaciones aleatorias (LLC, CCL, LCL, CLC, CLL y LCC), sin que en ninguna combinación se repita tres veces la misma duración. De la misma manera que en la prueba de patrones de frecuencia, se recomienda que esta prueba sea aplicada entre los 20 a $50 \mathrm{~dB}$ $\mathrm{SL}, 020$ a $50 \mathrm{~dB}$ sobre el promedio tonal puro $(\mathrm{PTP})^{4}$ y los resultados se expresan igualmente en porcentaje de respuestas correctas. En esta prueba también son aceptadas las modalidades de respuesta mediante tarareo los tonos indicando figuras que representen el sonido escuchado ${ }^{46}$.

Debido a que esta prueba originalmente es extensa y utiliza el mismo paradigma de evaluación que la prueba de patrones de frecuencia, se puede simplificar siguiendo los mismos criterios ${ }^{45,46}$. Se ha demostrado que posee una sensibilidad de $86 \%$ para detectar lesiones en el SNAC y una especificidad de $92 \%$. Es más resistente a los efectos de las lesiones cocleares que la prueba de patrones de frecuencias, debido a que no es altamente dependiente de una buena discriminación frecuencial ${ }^{50}$.

\section{CONSIDERACIONES CLÍNICAS DE LAS PRUEBAS DE PATRONES}

Tanto la prueba de patrones de frecuencia como la de duración, han demostrado tener buena sensibilidad y especificidad para detectar lesiones en el SNAC, $y$, por ende, para diagnosticar alteraciones del procesamiento temporal, lesiones cerebrales y algunas lesiones a nivel de tronco cerebral. Estas pruebas son además sensibles a lesiones del cuerpo calloso y disfunciones interhemisféricas ${ }^{49,50}$. Sin embargo, se ha demostrado que existen lesiones que solo son identificables con la prueba de patrones de duración y no con la de frecuencia y viceversa, por lo que a pesar de compartir los mismos sustratos neuroanatómicos, ambas pruebas tendrían distintos procesos subyacentes ${ }^{45}$. Por este motivo, se sugiere considerar las pruebas de patrones como evaluaciones complementarias. Cabe destacar que desde 1997, se comenzó a comercializar la prueba de patrones de frecuencia y de duración por la empresa norteamericana Auditec ${ }^{\circledR}$ pero con algunas diferencias ${ }^{51}$. La primera difiere principalmente en las frecuencias de los estímulos utilizados (880 y $1.430 \mathrm{~Hz}$ ) y en la introducción de dos nuevas versiones: una versión para adultos, en donde cada tono tiene una duración de $300 \mathrm{~ms}$ y un ITI de $6 \mathrm{~s}$, y una versión para niños, en donde cada estímulo tiene una duración de $500 \mathrm{~ms}$ y un ITI de 9 s. Por otro lado, la versión de la prueba de patrones de duración propuesta por Auditec ${ }^{\circledR}$ tiene las mismas características que la prueba original ${ }^{51}$.

Ambas pruebas han sido normadas para distintos grupos etarios y hablantes de distintos idiomas (Tabla 2). Este último aspecto es especialmente relevante ya que podrían existir diferencias de rendimiento en estas pruebas dependiendo de la lengua materna del grupo estudiado, teniendo en consideración que algunos idiomas requieren, por ejemplo, de una mayor habilidad de reconocimiento de patrones suprasegmentales del habla, específicamente de las variaciones de frecuencia, como es el caso de las lenguas tonales (principalmente las lenguas orientales $)^{52}$. En este tipo de poblaciones, un proceso normal de desarrollo del lenguaje implicaría por sí mismo un mayor entrenamiento auditivo en relación a otros idiomas. Además, se debe tener presente que la maduración del SNAC influye directamente en los valores normativos de poblaciones pediátricas. Una serie de cambios morfológicos asociados a la edad influencian las manifestaciones auditivas conductuales en los niños, siendo el más importante de ellos la mielinización ${ }^{2}$. Mientras que en algunas zonas del tronco cerebral la mielinización se encontraría completa alrededor del primer año de vida, la mayoría de las áreas corticales, además del cuerpo calloso, no terminarían este proceso hasta la edad adulta ${ }^{53}$. Por este motivo, algunos autores recomiendan que la aplicación de estas pruebas sea a partir de los ocho años de edad ${ }^{46}$, aunque Bellis ${ }^{2}$ propone que es posible aplicar la prueba de patrones de frecuencia desde los siete años y la prueba de patrones de duración a partir de los 9 años.

Cabe destacar que en los porcentajes presentados en la Tabla 2, corresponden a puntajes de corte obtenidos en poblaciones con audición periférica normal. Seis estudios revisados ${ }^{56-61}$ incluyeron a sujetos con PTP igual o inferior a $20 \mathrm{~dB}$ HL en audiometría tonal, curvas $\mathrm{A}$ bilaterales en la timpanometría y 


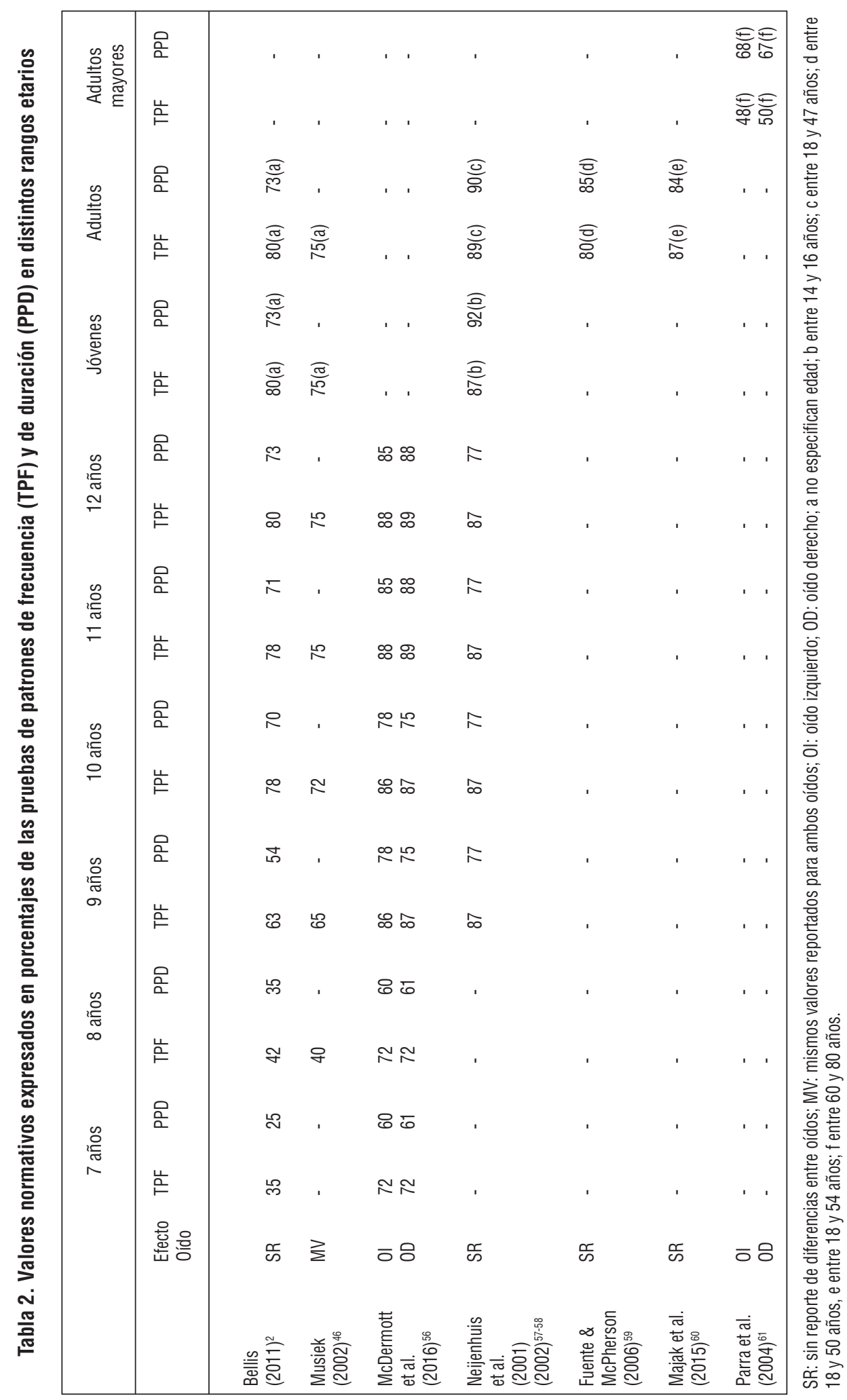


reflejos acústicos ipsi y contralaterales presentes en las frecuencias $0,5,1$ y $2 \mathrm{kHz}$. En solo uno de estos artículos ${ }^{61}$ se incluyó además la logoaudiometría con porcentajes de reconocimiento de la palabra iguales o superiores a $88 \%$ para incluir a los sujetos de estudio. Dos de los autores ${ }^{2,46}$ no especifican cómo se determinó la indemnidad auditiva periférica. En ninguno de los estudios revisados se encontraron diferencias significativas entre oídos, por cual cinco de los artículos revisados ${ }^{2,57-60}$ tomaron los valores obtenidos en el oído derecho más los del oído izquierdo para extraer un solo valor normativo válido para ambos.

Como se explicó anteriormente, la capacidad de obtener un rendimiento adecuado en las pruebas de patrones auditivos, está determinada por la indemnidad anatómica y fisiológica de ambos hemisferios cerebrales y del cuerpo calloso. En la Figura 3-A, se ilustra la responsabilidad de cada oído, de cada hemisferio y del cuerpo calloso respecto al procesamiento de los estímulos de las pruebas de patrones. La información respecto al reconocimiento del contorno debe ser procesada en el hemisferio derecho, y luego, debe pasar vía cuerpo calloso al hemisferio izquierdo en donde la señal puede ser etiquetada lingüísticamente. Una lesión o disfunción en cualquiera de estas dos estructuras, resultará generalmente en déficits bi- laterales ${ }^{45}$, sin que existan diferencias significativas entre oído izquierdo y derecho en ninguna de las dos pruebas ${ }^{50,54,55}$. Sin embargo, es posible identificar un sitio de lesión específico, dependiendo de las dificultades y de las respuestas que el sujeto examinado presente durante la prueba.

Cuando el sujeto evaluado tiene dificultades para verbalizar el orden de los estímulos percibidos, se le debe solicitar que vocalice, tararee o imite el patrón escuchado. En los casos donde encontramos un desempeño deficiente bajo la modalidad de respuesta verbal, pero un buen rendimiento mediante la imitación, probablemente estemos frente a la presencia de una disfunción en la transferencia interhemisférica hacia el hemisferio izquierdo (Figura 3-B), o una disfunción en el mismo hemisferio izquierdo (Figura 3-C) ${ }^{6}$. En ambos casos, no se trataría de una alteración auditiva propiamente tal, ya que la percepción y la capacidad de procesar los estímulos según su orden de aparición, estaría conservada, lo que quedaría evidenciado por las respuestas correctas mediante la imitación de las secuencias. Esto se ha demostrado en una serie de estudios de pacientes con cerebros divididos o con secciones quirúrgicas del cuerpo calloso ${ }^{11,49,50,62,63}$. Además, se ha demostrado que gran parte de los pacientes

Figura 3. Topodiagnóstico de lesiones mediante las pruebas de patrones de frecuencia y duración.

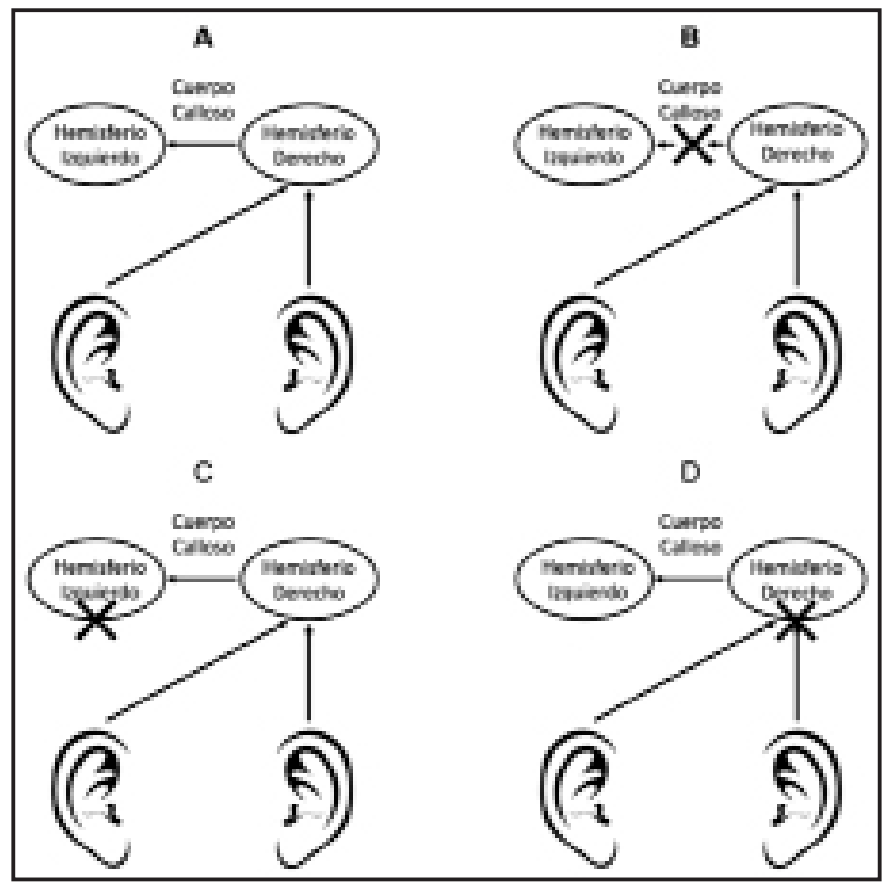


afásicos con lesiones documentadas en el hemisferio izquierdo mantienen intacta su capacidad para imitar y/o cantar, aun cuando el habla se encuentre comprometida ${ }^{64}$.

Aunque inicialmente la administración de ambas pruebas fue descrita mediante la utilización de auriculares para que la presentación de estímulos se pudiese realizar de manera monoaural ${ }^{45}$, se han realizado estudios en los cuales la presentación de los estímulos se ha efectuado de manera binaural65, e incluso mediante la utilización de sistemas de campo libre ${ }^{66}$. Estos estudios no reportan diferencias significativas en comparación a la presentación monoaural, al menos en sujetos con audición periférica y central indemnes, ya que tampoco existen diferencias entre oído derecho e izquierdo. Esto último sería de gran utilidad para la evaluación del ordenamiento temporal en sujetos que utilizan ayudas auditivas como audífonos e implantes cocleares.

En aquellos casos en los cuales exista algún tipo de daño en el hemisferio derecho, el reconocimiento del contorno, la percepción de la altura tonal y el reconocimiento de los patrones se encontrarían alterado $\mathrm{s}^{21}$. Por lo tanto, independiente de la modalidad de la respuesta, ya sea verbal 0 mediante imitación, las pruebas se encontrarán igualmente alteradas (Figura 3-D). Esto se ha demostrado en estudios de pacientes con cortectomía temporal tanto izquierda como derecha, reafirmando que el hemisferio derecho, representa la melodía y el contorno melódico en términos generales ${ }^{67}$. En estudios realizados en pacientes con amusia congénita, se han detectado anomalías en el giro frontal inferior y en la corteza auditiva del hemisferio derecho $0^{68-70}$. El término amusia se refiere al déficit específico de la percepción musi$\mathrm{cal}$, sin afectar al componente motor musical (voz cantada 0 ejecución de un instrumento musical), ni tampoco a los procesos cognitivos o emotivos que subyacen a la percepción musical ${ }^{71}$, afectando principalmente el procesamiento de la altura tonal ${ }^{72}$ y a la memoria melódica de corto plazo ${ }^{73}$. Cabe destacar que, aunque el principal análisis de estos aspectos recae en el hemisferio derecho, una menor parte de este procesamiento también ocurriría en el hemisferio izquierdo ${ }^{74}$.

Un último alcance tiene relación con los niveles de pérdida auditiva a los cuales se pueden realizar estas dos pruebas. Si bien se ha mencionado anteriormente que tanto la prueba de patrones de frecuencia como de duración no se ven afectadas por hipoacusias leves y moderadas ${ }^{49,50}$, esto no sería del todo cierto para hipoacusias severas 0 profundas. Se ha descrito que en hipoacusias sensorioneurales que superan los $60 \mathrm{~dB}$ se producirían alteraciones principalmente a nivel de mecánica coclear, que impedirían un adecuado procesamiento frecuencial y de algunos rasgos de la duración de los estímulos ${ }^{32}$. Por este motivo, se debe cautelar la interpretación de los resultados cuando se evalúan pacientes con pérdidas auditivas severas o mayores, teniendo en cuenta que ya estarían influyendo factores periféricos y no se trataría simplemente de un reflejo del funcionamiento del SNAC.

\section{CONCLUSIONES}

Las habilidades de procesamiento temporal subyacen al resto de las habilidades auditivas. Dentro de los aspectos temporales principales se encuentra el ordenamiento auditivo temporal, que es fundamental para una gran variedad de actividades cotidianas, de las que destaca principalmente la percepción adecuada del habla y del lenguaje. Los audiólogos deberían considerar incluir alguna prueba de procesamiento temporal dentro de la batería de evaluación clínica para determinar la integridad del SNAC y para identificar posibles déficits funcionales. Las pruebas de patrones de frecuencia y de duración han sido ampliamente utilizadas durante décadas para la evaluación de los aspectos temporales de la audición, específicamente del ordenamiento auditivo temporal, pudiendo detectar lesiones cerebrales y lesiones a nivel de tronco cerebral. Ambas cuentan con una buena sensibilidad y especificidad para detectar lesiones en el SNAC, son relativamente resistentes a lesiones auditivas periféricas y permiten realizar un topodiagnóstico de la lesión.

Además de ser de rápida y fácil aplicación e interpretación, ambas pruebas cuentan con valores normativos establecidos para distintos rangos etarios. Se recomienda ampliamente su utilización en conjunto con otras pruebas que han demostrado valores de sensibilidad y especificidad similares y que evalúen el 
resto de las habilidades auditivas como, por ejemplo, el Masking Level Difference (MLD), dígitos dicóticos (DD), pruebas de habla filtrada, entre otras. Sin embargo, debido a la variabilidad observada en los distintos estudios revisados, se sugiere que cada clínico establezca sus propios valores normativos locales,

\section{BIBLIOGRAFÍA}

1. American Speech-Language-Hearing Association. (2005). Technical Report: (Central) Auditory Processing Disorders. Obtenido de American Speech-Language-Hearing Association: http:// www.asha.org/policy/TR2005-00043.htm.

2. BeluIS, T. (2011). Assessment and Management of Central Auditory Processing Disorders in the Educational Setting: From Science to Practice (Segunda ed.). San Diego, CA: Plural Publishing.

3. Chermak, G., \& Musiek, F. (1997). Central auditory processing disorders: New perspectives. San Diego, CA: Singular.

4. Brooke, J. (2014). Temporal Processing Tests. En F. Musiek, \& G. Chermak, Handbook of Central Auditory Processing Disorder: Auditory Neuroscience and Diagnosis (Segunda ed., pp. 405-434). San Diego, CA: Plural Publishing.

5. HIRSH, I. Auditory perception of temporal order. $J$ Acoust Soc Am 1959; 31(6): 759-67.

6. Pinheiro, M., \& Musiek, F. (1985). Assessment of central auditory dysfunction: Foundations and clinical correlates. Baltimore, MD: Williams and Wilkins.

7. HIRSH, I. (1967). Information processing in input channels for speech and language: The significance of serial order on stimuli. En. In C. Milliman, \& F. Darley, Brain mechanisms underlying speech and language (pp. 22-38). New York, NY: Grune and Stratt.

8. NefF, W. (1961). Neural mechanisms of auditory discrimination. In W. Rosenblish, Sensory communication (pp. 259-277). Cambridge, MA: MIT Press.

9. Pichora-Fuller, M., \& Souza, P. Effects of aging on auditory processing of speech. Int $J$ Audiol 2003; 42(Suppl 2): 11-6.

10. Onoda, R., Pereira, L., \& Guilherme, A. Reconhecimento de padrão temporal e escuta dicótica em descendentes de japoneses, falantes e não-falantes da língua japonesa. Rev Bras especialmente en población pediátrica, donde la lengua materna, escolaridad, ambiente sociocultural, presencia de trastornos del lenguaje, alteraciones del aprendizaje, déficit atencional con hiperactividad u otros trastornos del desarrollo, podrían influir en los resultados de la prueba.

Otorrinolaringol 2006; 72(6): 737-46.

11. Musiek, F., Pinheiro, M., \& Wilson, D. Auditory Pattern Perception in 'Split Brain' Patients. Arch Otolaryngol 1980; 106(10): 610-2.

12. Halperin, Y., Nachshon, I., \& Carmon, A. (1973). Shift of ear superiority in dichotic listening of temporally patterned nonverbal stimuli. J Acoust Soc Am 1973; 53(1): 46-50.

13. EFron, R. The effect of handedness on the perception of simultaneity and temporal order. Brain Research 1963; 86(1): 261-84.

14. KImURA, D. Left-right differences in the perception of melodies. Q J Exp Psychol 1964; 16(4): 355-8.

15. SwISHER, L., \& HIRSH, I. Brain damage and the ordering of two temporally successive stimuli. Neuropsychologica 1972; 10(2): 137-52.

16. Pinheiro, M., \& Ptacek, P. (1971). Reversals in the perception of noise and tone patterns. J Acoust Soc Am 1971; 49(6B): 1178-782.

17. JeRGER, J. (1997). Functional asymmetries in the auditory system. Ann Otol Rhinol Laryngol Suppl 1997; 168: 23-30.

18. Wioland, N., Rudolf, G., Metz-Lutz, M., Mutschler, V., \& MarescauX, C. Cerebral correlates of hemispheric lateralization during a pitch discrimination task: an ERP study in dichotic situation. Clin Neurophysiol 1999; 110(3): 516-23.

19. Blumstein, S., \& Cooper, W. Hemispheric processing of intonation contours. Cortex 1974; 10(2): 146-58.

20. NeBES, R. Handedness and the perception of partwhole relationship. Cortex 1971; 7(4): 350-6.

21. Joanette, Y., Ansaldo, I., Kahlaoui, K., Côté, H., Abusamra, V., Ferreres, A., \& Roch-Lecours, A. Impacto de las lesiones del hemisferio derecho sobre las habilidades lingüísticas: perspectivas teórica y clínica. Rev Neuro/ 2008; 46(8): 481-8.

22. Boas, L., Muniz, L., Caldas, S., \& Gouveia, M. Desempenho do processamento auditivo temporal em uma população de cegos. Braz J Otorhinolaryngol 2011; 77(4): 504-9. 
23. Bryden, M., Hecaen, H., \& De Agostini, M. Patterns of cerebral organization. Brain Lang 1983; 20(2): 249-62.

24. Goodglass, H., \& QuADFASEL, F. Language laterality in left-handed aphasics. Brain 1954; 77(4): 521-48.

25. GaZZANIGA, M., \& SperRY, R. Language after section of the cerebral commissures. Brain 1967; 90(1): 131-48.

26. SnelL, R. (2007). Neuroanatomía Clínica (Sexta ed.). Buenos Aires: Médica Panamericana.

27. Pulvermüller, F. (2002). The Neuroscience of Language. On Brain Circuits of Words and Serial Order. New York, NY: Cambridge University Press.

28. Brownell, H., Simpson, T., Bihrle, A., Potter, H., \& Gardner, H. Appreciation of metaphoric alternative word meanings by left and right braindamaged patients. Neuropsychologia 1990; 28(4): 375-83.

29. Le Blanc, B., \& Joanette, Y. Unconstrained oral naming in left- and righthemisphere-damaged patients: an analysis of naturalistic semantic strategies. Brain Lang 1996; 55(1): 42-5.

30. Vanhalle, C., Lemieux, S., Joubert, S., Goulet, P., Ska, B., \& JoAnEtTE, Y. Processing of speech acts by right hemisphere brain-damaged patients: an ecological approach. Aphasiology 2000; 14(11): 1127-41.

31. Joanette, Y., Goulet, P., \& Hannequin, H. (1990). Right Hemisphere and Verbal Communication. New York, NY: Springer-Verlag.

32. Gelfand, S. (2010). Hearing: An introduction to psychological and physiological acoustics (Quinta ed.). London: Informa Healthcare.

33. PHILLIPS, D. Auditory gap detection, perceptual channels and temporal resolution in speech perception. J Am Acad Audiol 1999; 10(6): 343-54.

34. Berwanger, D., Wittmann, M., von Steinbüchel, N., \& Von Suchodoletz, W. Measurement of temporalorder judgment in children. Acta Neurobiol Exp (Wars) 2004; 64(3): 387-94.

35. HiRSh, I., \& SherRick, C. Perceived order in different sense modalities. J Exp Psychol 1961; 62: 423-32.

36. Tallal, P., Milleer, S., \& Fitch, R. Neurobiological basis of speech: a case for the preeminence of temporal processing. Ann N Y Acad Sci 1993; 14(682): 27-47.

37. Farmer, M., \& Klein, R. The evidence for a temporal processing deficit linked to dyslexia: A review. Psychon Bull Rev 1995; 2(4): 460-93.
38. Salvi, R., Lockwood, A., Frisina, R., Coad, M., WACK, D., \& FRISINA, D. PET imaging of the normal human auditory system: responses to speech in quiet and in background noise. Hear Res 2002; 170(1-2): 96-106.

39. Poremba, A., Saunders, R., Crane, A., Cook, M., SoKoloff, L., \& MISHKIN, M. Functional mapping of the primate auditory system. Science 2003; 299(5606): 568-72.

40. Warren, R., \& Obusek, C. Identification of temporal order within auditory sequences. Percept Psychophys 1972; 12(1): 86-90.

41. Leshowitz, B., \& Hanzi, R. Auditory pattern discrimination in the absence of spectral cues. J Acoust Soc Am 1972; 52(1A): 166-76.

42. Pinheiro, M. (1977). Tests of central auditory function in children with learning disabilities. In R. Keith, Central auditory dysfunction (pp. 223-256). New York, NY: Grune \& Stratton.

43. Emanuel, D. The auditory processing battery: Survey of common practices. J Am Acad Audiol 2002; 13(2): 93-117.

44. Emanuel, D., Ficca, K., \& Korczak. Survey of the diagnosis and management of auditory processing disorders. Am J Audiol 2011; 20(1): 48-60.

45. Musiek, F. Frequency (pitch) and duration pattern tests. J Am Acad Audiol 1994; 5(4): 265-86.

46. Musiek, F. The frequency pattern test: A guide. Hear J 2002; 55(6): 58.

47. Musiek, F., \& GEURKINK, N. Auditory perceptual problems in children: considerations for the otolaryngologist and audiologist. Laryngoscope 1980; 90(6): 962-71.

48. Musiek, F., Geurkink, N., \& Keitel, S. Test battery assessment of auditory perceptual dysfunction in children. Laryngoscope 1982; 92(3): 251-7.

49. Musiek, F., \& Pinheiro, M. Frequency patterns in cochlear, brainstem and cerebral lesions. Audiology 1987; 26(2): 79-88.

50. Musiek, F., Baran, J., \& Pinheiro, M. Duration pattern recognition in normal subjects and patients with cerebral and cochlear lesions. Audiology 1990; 29(6): 304-13.

51. Auditec. (1997). Evaluation manual of pitch pattern sequence and duration pattern sequence. Missouri: Auditec Inc.

52. Haspelmath, M., Dryer, M., Gil, D., \& Comrie, B. (2005). The World Atlas of Languages Structures. Oxford: Oxford University Press. 
53. Pujol, J., Vendrell, P., Junqué, C., Marti-Vilalta, J., \& CAPDEVILA, A. When does human brain development end? Evidence of corpus callosum growth up to adulthood. Ann Neurol 1993; 34(1): 71-5.

54. Pinheiro, M., \& Godbey, N. (1992). Perceptual Reversals of Temporal Patterns with Co-Varying Acoustic Cues. Annual meeting of the American Speech and Hearing Association. Detroit, MI.

55. Pinheiro, M. Auditory pattern perception in patients with right and left hemisphere lesions. Ohio J Speech Hear 1976; 12(1): 9-20.

56. McDermott, E., Smart, J., Bolano, J., Bragg, L., Colon, T., Hanson, E., Kelly, A. Assessing Auditory Processing Abilities in Typically Developing School-Aged Children. J Am Acad Audiol 2016; 27(2): 72-84.

57. Neijenhuis, K., Stollman, M., Snik, A., \& Van der BROEK, P. Development of a central auditory test battery for adults. Audiology 2001; 40(2): 69-77.

58. Neijenhuis, K., Snik, A., Priester, G., van Kordenoordt, S., \& van den Broek, P. Age effects and normative data on a Dutch test battery for auditory processing disorders. Int J Audiol 2002; 41(6): 334-46.

59. Fuente, A., \& McPherson, B. Auditory processing tests for Spanish-speaking adults: an initial study. Int J Audiol 2006; 45(11): 645-59.

60. Majak, J., Zamysłowska-Szmytke, E., \& Rajkowska, E. Auditory temporal processing tests - Normative data for Polish-speaking adults. Med Pr 2015; 66(2): 145-52.

61. Parra, V., Iorio, M., Mizahi, M., \& Baraldi, G. Frequency and duration patterns tests in elderly people with normal hearing sensitivity. Rev Bras Otorrinolaringol 2004; 7(4): 517-23.

62. Baran, J., Musiek, F., \& Reeves, A. Central auditory function following anterior sectioning of the corpus callosum. Ear Hear 1986; 7(6): 359-62.

63. Musiek, F., \& Reeves, A. Asymmetries of the auditory areas of the cerebrum. J Am Acad Audiol 1990; 1(4): 240-5.

64. Sмітн, A. Speech and other functions after left (dominant) hemispherectomy. J Neurol Neurosurg Psychiatry 1966; 29(5): 467-71.
65. Ribas, C., Vieira, A., Figueiredo, A., \& Guida, $H$. Pitch pattern sequence and duration pattern tests in Brazil: literature review. Rev CEFAC 2014; 16(1): 283-92.

66. Frederigue-Lopes, N., Bevilacqua, M., Sameshima, K., \& CostA, O. Desempenho de crianças normais em testes temporais auditivos em campo livre. Pró-Fono R Atual Cient 2010; 22(2): 83-8.

67. Peretz, I. Processing of local and global musical information by unilateral brain-damaged patients. Brain 1990; 113(Pt 4): 1185-205.

68. Hyde, K., Lerch, J., Zatorre, R., Griffiths, T., Evans, A., \& Peretz, I. Cortical thickness in congenital amusia: when less is better than more. $J$ Neurosci 2007; 27(47): 13028-32.

69. Hyde, K., Zatorre, R., \& Peretz, I. Functional MRI evidence of an abnormal neural network for pitch processing in congenital amusia. Cereb Cortex 2011; 21(2): 292-229.

70. Hyde, K., Zatorre, R., Griffiths, T., Lerch, J., \& PeRETZ, I. Morphometry of the amusic brain: a two-site study. Brain 2006; 129(Pt 10): 2562-70.

71. WiPE, B., Kuroiwa, M., \& DÉlano, P. Trastornos de la percepción musical. Rev Otorrinolaringol Cir Cabeza Cuello 2013; 73(2): 189-99.

72. Chen, J., \& Yuan, J. The neural causes of congenital amusia. J Neurosci 2016; 36(30): 803-4.

73. Leveque, Y., Fauvel, B., Groussard, M., Caclin, A., Albouy, P., Platel, H., \& Tillmann, B. Altered intrinsic connectivity of the auditory cortex in congenital amusia. J Neurophysio/2016; 116(1): 88-97.

74. Patterson, R., Uppenkamp, S., Johnsrude, I., \& GRIFFITHS, T. The processing of temporal pitch and melody information in auditory cortex. Neuron 2002; 36(4): 767-76.

75. Musiek, F., \& Chermak, G. (2015). Psychophysical and behavioral peripheral and central auditory tests. En M. Aminoff, F. Boller, \& D. Swaab, Handbook of Clinical Neurology: The Human Auditory System Fundamental Organization and Clinical Disorders (Vol. 129, pp. 313-332). Waltham, MA: Elsevier B.V.

\section{Dirección: Anthony Marcotti F. Facultad de Ciencias de la Salud, Universidad de las Américas E mail: amarcotti @udla.cl}

